\title{
Numerical Investigation of Head-on Binary Drop Collisions in a Dynamically Inert Environment
}

\author{
M. D. Saroka ${ }^{1}$, N. Ashgriz ${ }^{2 \dagger}$ and M. Movassat ${ }^{2}$ \\ ${ }^{1}$ United Technologies Research Center, 411 Silver Lane, MS 129-1, East Hartford, CT, USA 06108 \\ ${ }^{2}$ Mechanical and Industrial Engineering Department, University of Toronto, Toronto, \\ Ontario, Canada M5S $3 G 8$
}

†Corresponding Author Email: Ashgriz@mie.utoronto.ca

(Received December 22, 2009; accepted July 13, 2010)

\begin{abstract}
The results of three-dimensional numerical simulations of drop collisions without the effect of a surrounding environment are presented. The numerical model is based on an Eulerian, finite-difference, Volume-of-Fluid method. Surface tension is included using the Continuum Surface Force method. Head-on collisions using equal size drops with three different fluid properties of water, mercury and tetradecane are presented. Various drop diameters ranging from $200 \mu \mathrm{m}$ to $5 \mathrm{~mm}$ are considered. A separation criterion based upon deformation data is found. The lower critical Weber numbers are found for mercury and water drops while tetradecane drops did not result in separation for the range of Weber numbers considered. The effect of Reynolds number is investigated and regions of permanent coalescence and separation are plotted in the Weber-Reynolds number plane. The role of viscosity and its effect on dissipation is also investigated. Finally, the validity of the assumptions made in some of the collision models is assessed.
\end{abstract}

Keywords: Drop collision, Drop coalescence, Drop dynamics, Volume of fluid method.

\section{INTRODUCTION}

Drop collision studies are relevant to a broad spectrum of fields ranging from large scales such as astrophysics and meteorology, to much smaller scales as seen in aerosols and nuclear physics. Due to the complex nature of colliding drops, the majority of previously published works have been restricted to experimental investigations and phenomenological analysis (Jayarante and Mason 1964; Gunn 1965; Ryley and Bennett-Cowell 1967; Adam et al. 1968; Park 1970; Whepdale and List 1971; Brazier-Smith et al. 1972; Bradley and Stow 1978; Bradley and Stow 1979; Low and List 1982a; Low and List 1982b; Arkhipov et al. 1983; Ashgriz and Givi 1987; Brenn and Frohn 1989; Ashgriz and Givi 1989; Ashgriz and Poo 1990; Jiang et al. 1992; Qian and Law 1997; Menchaca-Roca et al. 1997; Brenn et al. 1997; Guido and Simeone 1998; Orme 1997; Estrade et al. 1999; Willis and Orme 2000; $\mathrm{Hu}$ et al. 2000; Brenn et al. 2001; Post and Abraham 2002; Willis and More 2003; Roisman 2004; Leal 2004; Gao et al. 2005; Ko and Ryou 2005; Yoon et al. 2005; Baldessari and Leal 2006). Experimental observations suggest that there are five major types of outcome when two liquid masses collide in a gaseous environment. They are bouncing, partial coalescence, coalescence, separation, and shattering. In a bouncing collision, contact of drop surfaces is prevented by the intervening gas film, and drops bounce apart without any mass exchange. A partial coalescence collision happens only for drops with very large size difference and very small velocities. The small drop attaches to the big drop and flows into the big drop because of the pressure difference between them. Before the small drop is completely absorbed by the large drop, the surface tension cuts off the bridge and a secondary drop is generated. Coalescence collisions refer to the collisions in which two drops permanently combine and generate one single drop. A separation collision is one in which drops coalesce temporarily and later separate into one single string of two or more drops of various sizes. A shattering collision, which is the characteristic of high relative velocity collision, is one in which the collided drops disintegrate into a cluster of many liquid fragments shortly after the collision. These five types are only the most general ones and more sub-categories within them can be defined if other factors, such as number of drops generated, mass transfer rate or shape relaxation rate, are taken into account.

The major objective of drop collision studies has been to develop models and correlations for various types of collision outcomes in terms of some initial conditions of the two drops. However, a limited number of quantitative models exist at the present time. These models can be divided into three categories. In the first category, the onset of breakup after the collision is modeled based on comparing the angular momentum of 
the collided pair, which pulls the drops apart, and the surface tension forces, which hold the drops together. These models are exemplified by the works of Park (1970), Brazier-Smith et al. (1972), Arkhipov, et al. (1983), Brenn et al. (2001), and Post and Abraham (2002). In the head-on collision of two drops - the topic of the present work - the angular momentum does not play an important role. Therefore, these models cannot explain the onset of separation for the head-on collisions. In the second category, the breakup is modeled based on comparing the total energy before and after the collision. The total energy includes kinetic, surface, and dissipation energies. Certain assumptions are made for the shape of the drop after the collision and for the dissipated energy. These models are exemplified by those of Jiang et al. (1992), Qian and Law (1997), and Willis and Orme (2000, 2003). And in the third category, some capillary instability criterion along with the energy consideration is used to predict the breakup. These models are exemplified by those of Ashgriz and Poo (1990) and Brenn et al. (1997).

The objective of the present paper is to gain further insight on the head-on collision dynamics of two drops and to provide a critical evaluation of above mentioned models using direct numerical simulation. The evolutionary behavior of the head-on drop collisions without the effect of the surrounding environment is considered. By not modeling the surrounding atmosphere, the effects due to the internal flow patterns and trapped gas between the approaching drops are removed.

\section{NUMERICAL METHODOLOGY}

\subsection{Review of the Previous Work}

Nobari et al. (1996) used the front-tracking method developed by Unverdi and Tryggvason (1992) to study the head-on collisions of equal sized drops. In their method, the governing equations are solved both inside and outside the drops, thus including the effect of the surrounding environment. By applying a body force to accelerate the drops toward each other both bouncing and coalescence were investigated. The presence of a double interface always resulted in bouncing, while coalescence was modeled by artificially rupturing the interfaces at prescribed rupture times.

Their results indicated that different rupture times result in different final outcomes for similar collisions. They could not predict the boundary between the coalescence and separation without an empirical input for the rupture time. The rupture times were adjusted to obtain the experimental seperatoin conditions.

Rieber and Frohn (1995) modified their previous work with Schelkle et al. (1996) to model drop collisions. In their algorithm they computed the volumetric surface tension force based on a conservative discretization for the curvature computation based on the work of Lafaurie et al. (1994). Using this technique, they performed a brief investigation of equal sized binary water drop collisions occurring in air. They considered Weber numbers between the range of 19 and 59, while maintaining a constant Reynolds number of 5000 and numerically computed the boundary between coalescence and separation (parameters are defined in the nomenclature). A two dimensional simulation of drop collision was conducted by Poo and Ashgriz (1990) using a volume of fluid method (Asgriz and Poo 1990). Since two dimensional drops do not provide the proper surface energy, the physically observed reflexive action could not be simulated with such models. Mashayek et al. (2003) studied the coalescence collision of two liquid drops using a Galerkin finite element method in conjunction with a spine-flux method for the free surface tracking (Mashayek and Ashgriz 1995). The effects of Reynolds number, impact velocity, drop size ratio, and internal circulation on the coalescence process was investigated. Only the coalescence collisions were studied and separation conditions were not reached.

Morozumi et al. (2005) conducted a numerical study to obtain a criterion between permanent coalescence and separation in head-on binary drop collisions. They used a Lagragian finite-element method to model the problem. They found that the ratio of the maximum length of the coalesced droplet to the initial diameter of the droplets is about 2.85 , beyond which the coalesced drop will breakup. Finally, Nikolopoulos et al. (2009) conducted an axis-symmetric numerical study on collision of two equal size drops employing a VOF solver and provide detailed results about the size of the satellite droplets forming after collision.

\subsection{Method of Solution}

A numerical model based on an Eulerian, finitedifference, Volume-of-Fluid (VOF) method with surface tension effects is used (Bussmann et al. 1999; Hsu and Ashgriz 2004a; Hsu and Ashgriz 2004b). We consider two drops composed of the same fluid with constant viscosity, $\mu$, density, $\rho$, and surface tension coefficient, $\sigma$. The flow inside the drops is solved using the standard conservation of mass and momentum equations:

$\nabla \cdot V=0$

$\rho \frac{\partial V}{\partial t}+\rho \nabla \cdot(V V)=-\nabla p+\mu \nabla^{2} V+F_{b}$

where $V$ is the velocity vector, $p$ is the pressure and $F_{b}$ is any body force per unit volume acting on the drops. At the free surface boundary condition, both mass and momentum are conserved. For drops surrounded by a less dense/viscous fluid or gas, the viscous stresses at the surface can be neglected. Additionally, assuming a constant surface tension coefficient allows the stress boundary condition to reduce to Laplace's equation:

$p_{s}=\sigma \kappa$

where, $p_{s}$ is the pressure jump across the surface and $\kappa$ is the surface curvature. The above governing equations are solved in the three-dimensional space using a two-step projection method as described by Kothe et al. (1991). In order to solve for the new velocity field, the momentum equation is divided into two steps 


$$
\begin{aligned}
& \frac{\tilde{V}-V^{n}}{\delta t}=-\nabla \cdot(V V)^{n}+\frac{\mu}{\rho^{n}} \nabla^{2} V^{n}+\frac{1}{\rho^{n}} F_{b}^{n} \\
& \frac{V^{n+1}-\tilde{V}}{\delta t}=-\frac{1}{\rho^{n}} \nabla p^{n+1}
\end{aligned}
$$

where $n$ and $n+1$ represent the previous and next time step, respectively. Equation (4) is solved for an intermediate velocity, $\tilde{V}$, by explicitly updating the

known velocity field, $V^{n}$, with the convective, viscous and surface tension effects. The surface tension effect is modeled using a form of the Continuum-Surface Force (CSF) method. In this method the surface tension effect is converted into a volumetric force for computational cells near the free surface. Although specific details are provided in the papers by Brackbill et al. (1992) and Bussmann et al. (1999), it is worth commenting that Bussmann et al. (1999) modified the original CSF method for use in simulations involving fluids in a vacuum. Their modification included the addition of a weighting function in the smoothed volumetric surface force calculation. This weighting function allows the volumetric surface force to be a nonzero, uniform body force in cells containing fluid and zero in empty cells. The time step restriction is computed based upon the smallest step size considering fluid advection, momentum transfer and capillary wave growth. Typically, the surface tension criterion is the most restrictive.

The second step of the method is used to project the intermediate velocity onto a divergence free field by combining the continuity Eq. (1) with Eq. (5). This results in the Poisson pressure equation which is symmetric and positive definite and is solved using an incomplete Cholesky conjugate gradient solver for the new pressure field, $p^{n+1}$. Lastly, the intermediate velocity field is updated using the new pressure field to give the new velocity, $V^{n+1}$ at the next time step. The free surfaces are resolved using the Volume-of-Fluid (VOF) method. VOF methods use a scalar defined as the volume fraction, $f$, which represents how much fluid is contained within a cell. If the cell is completely filled with fluid then $f=1$, while if the cell is completely void of fluid, $f=0$. For a partially filled cell (i.e. a surface cell) then $0<f<1$. Thus once the new velocity field is computed this information is used to determine the updated fluid fraction field at the next time step. However, in free surface problems, it is not sufficient to just solve for the fluid flow. The free surface must be evaluated in terms of both its new position and its orientation. This is done by using a 3D volume tracking method developed by Youngs (1984). In this methodology, polygonal planes are computed which match the volume fraction of a given cell. The orientation of the plane is then adjusted based upon the computed normal vector directed into the liquid phase.

\subsection{Simulation Setup}

Since only head-on collisions are considered in this investigation, planes of symmetry were used to reduce the amount of computational time and cost. Specifically, all simulations were modeled using onequarter profiles for each of the two drops (20 cells per drop radius) and two planes of symmetry. In order to cause the drops to collide, each drop was given a nonzero initial velocity. These individual drop velocities were subsequently used to compute the relative velocity, $V_{r}$. Various simulations were conducted for two drops composed of either water, mercury or tetradecane $\mathrm{C}_{14} \mathrm{H}_{30}$. Constant fluid properties were used (collisions between different fluids were not considered). Same size water drops with diameters of $D=300 \mu \mathrm{m}, 500 \mu \mathrm{m}, 700 \mu \mathrm{m}$ and $2 \mu \mathrm{mm}$ were simulated for the majority of the computations. However, during the investigation concerning the effect of Reynolds number, simulations for drop diameters of $200 \mu \mathrm{m}, 1 \mathrm{~mm}, 1.5 \mathrm{~mm}, 3 \mathrm{~mm}$ and $5 \mathrm{~mm}$ were also performed. All mercury and tetradecane collisions were for drops with a diameter of $300 \mu \mathrm{m}$. The fluid properties used are summarized in Table 1.

Table 1 Drop Fluid Properties

\begin{tabular}{|c|c|c|c|}
\hline Fluid & $\begin{array}{c}\text { Density } \\
{\left[\mathrm{kg} / \mathrm{m}^{3}\right]}\end{array}$ & $\begin{array}{c}\text { Kinematic } \\
\text { Viscosity } \\
{\left[\mathrm{m}^{2} / \mathrm{s}\right]}\end{array}$ & $\begin{array}{c}\text { Surface } \\
\text { Tension } \\
{[\mathrm{N} / \mathrm{m}]}\end{array}$ \\
\hline Water & 1000 & $1.0 \times 10^{-6}$ & 0.073 \\
\hline Mercury & 13550 & $1.2 \times 10^{-7}$ & 0.484 \\
\hline Tetradecane & 763 & $3.02 \times 10^{-6}$ & 0.027 \\
\hline
\end{tabular}

The droplet dynamics is described based on Weber and Reynolds numbers, defined as $W e=\rho V_{r}^{2} D / \sigma$ and $\operatorname{Re}=\rho V_{r} D / \mu$, respectively. Numerical simulations of drops colliding at high impact energies require a fine grid to resolve the flow in either the radial or axial direction. Therefore, collisions are limited by a maximum collision velocity beyond which a proper grid resolution could not be achieved. These velocities are as follows: For water drop collisions, relative velocities less than $3.8 \mathrm{~m} / \mathrm{s}, 2.7 \mathrm{~m} / \mathrm{s}, 2.3 \mathrm{~m} / \mathrm{s}$ and 1.6 $\mathrm{m} / \mathrm{s}$ were used for $300 \mu \mathrm{m}, 500 \mu \mathrm{m}, 700 \mu \mathrm{m}$ and $2 \mathrm{~mm}$ drops, respectively.
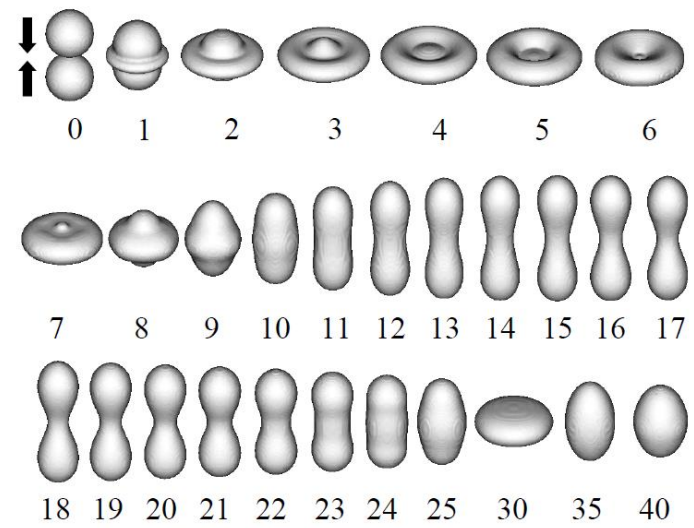

Fig. 1. Surface Evolution for a Binary Water Drop Collision with $\mathrm{We}=40$ and $\mathrm{Re}=936$. Droplet diameter of each drop is $300 \mu \mathrm{m}$, and their relative velocity is

$3.12 \mathrm{~m} / \mathrm{s}$. Collision times are equal to $(0.05 \mathrm{~ms}) i$, where $i=$ image number. 
For Mercury drop collisions, relative velocities less than $2.7 \mathrm{~m} / \mathrm{s}$, while for tetradecane drop collisions, relative velocities less than $11 \mathrm{~m} / \mathrm{s}$ were considered.

\section{RESULTS AND DISCUSSION}

We have conducted an extensive numerical simulation of head-on collisions of two drops. Figures 1 and 2 show typical surface evolutions for two different collisional outcomes. Figure 1 shows the evolution sequence for two $300 \mu \mathrm{m}$ water drops with a relative velocity of $3.12 \mathrm{~m} / \mathrm{s}$. This results in $\mathrm{We}=40$ and $R e=936$, with an outcome of permanent coalescence. This series of images is provided to illustrate the various surface configurations as the combined mass evolves toward a single spherical drop at $t>40$. Subsequently, as the impact velocity is increased to $3.76 \mathrm{~m} / \mathrm{s}$, the colliding drops will combine temporarily and eventually separate as shown in Fig. 2. This figure represents a collision between the same $300 \mu \mathrm{m}$ water drops as in Fig. 1, but with a relative velocity of $3.76 \mathrm{~m} / \mathrm{s}$, resulting in $W e=58$ and $R e=1127$.

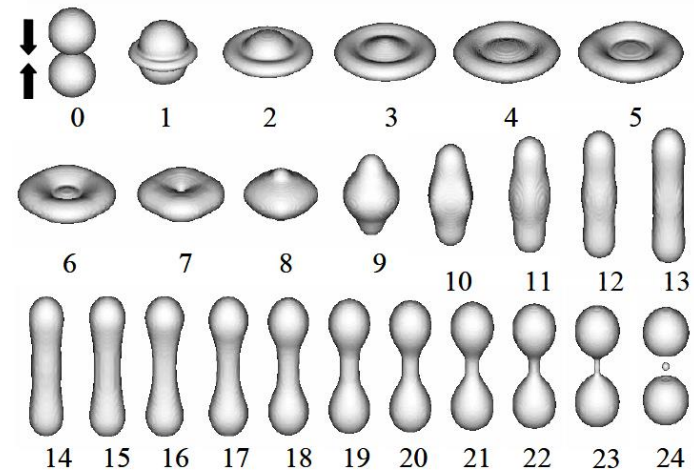

Fig. 2. Surface Evolution for a Binary Water Drop Collision with $\mathrm{We}=58$ and $\mathrm{Re}=1127$. Droplet diameter of each drop is $300 \mu \mathrm{m}$, and their relative velocity is

$3.76 \mathrm{~m} / \mathrm{s}$. Collision times are equal to $(0.05 \mathrm{~ms}) i$, where $i=$ image number.

We can divide the collision process into several phases. Snap shots of surface shapes during various phases of collision are shown in Fig. 3. The collision coordinate system is shown on the upper right corner of this figure. The $\mathrm{z}$-axis is referred to as the axial direction, whereas the flow in the $x-y$ plane is referred to as the radial direction. The following phases are defined for head-on binary drop collisions: (i) the collision phase: radial expansion and axial contraction $\left(0 \leq \mathrm{t} \leq \mathrm{t}_{1}\right)$; (ii) the reflex phase: radial contraction $\left(\mathrm{t}_{1}<\mathrm{t} \leq \mathrm{t}_{2}\right)$ and axial expansion $\left(\mathrm{t}_{1}<\mathrm{t} \leq \mathrm{t}_{4}\right)$; and (iii) the oscillation or the break up phase depending upon whether the final outcome is coalescence or separation $\left(t>t_{4}\right)$. In addition, time $t_{3}$ is defined as the time when the fluid flow has reversed its direction from radial to axial direction. Since this time reflects a change in the bulk direction of the internal flow, the corresponding shape of the drop at this point is provided. The collision phase (i) or the radial expansion is very similar to the spreading effect observed in drop impaction studies as is depicted in images 1-4 on Figs. 1 and 2. Once the fluid has reached its maximum deformation or $R_{\max }$, the combined mass begins to contract due to the surface tension forces as shown by images 5-8 on the figures. At this point, the flow is re-directed 90 degrees or in the axial direction. As the fluid continues to flow in this direction, the combined mass stretches or expands until a maximum deformation defined as $Z_{\max }$ is obtained. This process is shown as images 9-14 on each of the figures. Once $Z_{\max }$ occurs, the surface tension force pushes the fluid back toward the center and the mass begins to contract (phase iii) as shown in the remaining images. The last phase can consist of either an oscillatory behavior (images $15-40$ of Fig. 1) or separation (image 24 of Fig. 2).

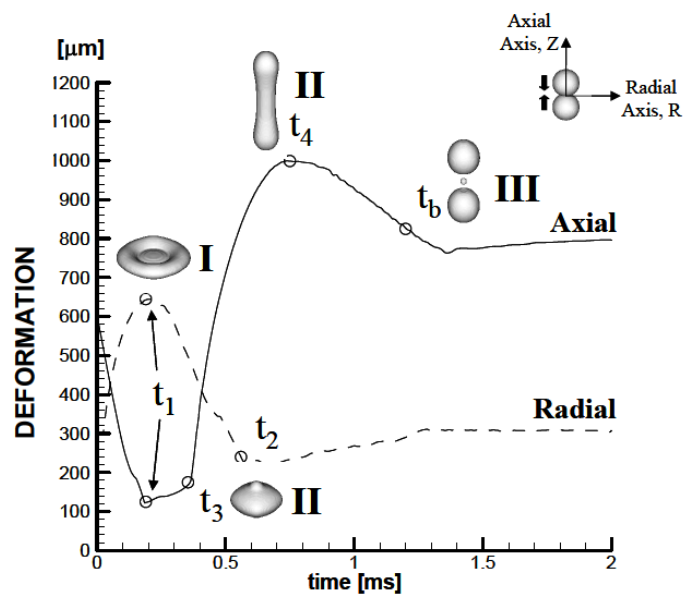

Fig. 3. Example of phase boundaries for two drop collision with $\mathrm{We}=58$ and $\mathrm{Re}=1127$. Solid line represents the axial deformation while the dashed line represents the radial deformation.

\subsection{Internal Flow Patterns}

Figure 4 shows both the internal pressure and velocity field for three specific times during the evolution of two $300 \mu \mathrm{m}$ water drops colliding at $\mathrm{We}=58$ and $R e=1127$. For each of the figures, the left side contains the pressure contours while the right side consists of velocity vectors and streamlines indicating the overall flow pattern.

Fig. 4a represents the flow just before beginning of the axial expansion period. A high pressure region at the center of the combined mass is formed due to the initial merging process. This redirects the flow outward or away from the center of the combined mass. Figure $4 \mathrm{~b}$ illustrates the flow pattern once the combined mass has reached its maximum deformation along the axial direction. At the next time step the flow near the bulbous ends is re-directed inward due to the surface tension effects. In Fig. 4b, the flow is still flowing outward and away from the center, while a high pressure region due to the surface curvature has developed at the top/bottom regions of the bulbous ends. Figure $4 \mathrm{c}$ represents the internal pattern when the combined mass has contracted and nearly separated.

An example illustrating the typical flow patterns during each stage of the evolution is provided in Fig. 5. In this figure, 11 images or slices represent the internal flow patterns for two $700 \mu \mathrm{m}$ water drops colliding at a relative velocity of $2.28 \mathrm{~m} / \mathrm{s}$ resulting in a $W e=50$ and $R e=1596$. As the two drops come into contact and 
collide with one another (image 1) a stagnation region develops at the center.
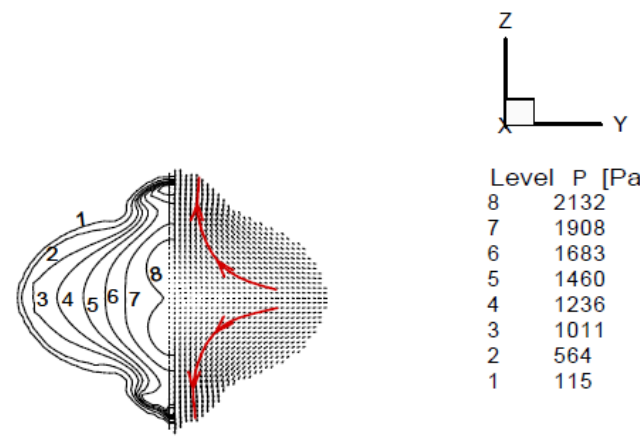

$\begin{array}{ll}\text { Level } P \text { P } & \text { [Pa] } \\ 8 & 2132 \\ 7 & 1908 \\ 6 & 1683 \\ 5 & 1460 \\ 4 & 1236 \\ 3 & 1011 \\ 2 & 564 \\ 1 & 115\end{array}$

(a)
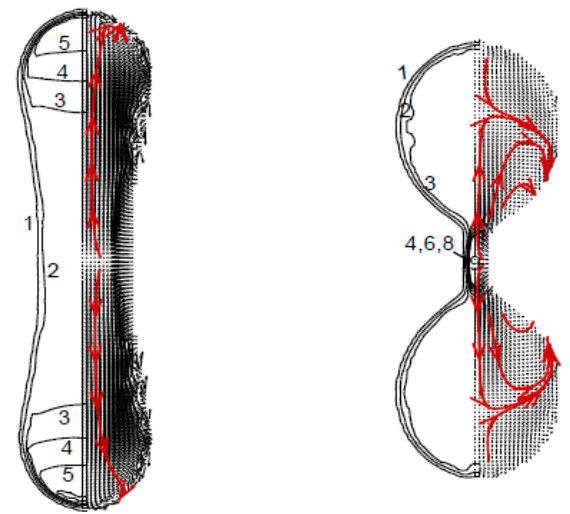

(b)

(c)

Fig. 4. Velocity and pressure fields inside an evolving drop (a) when kinetic energy is near its maximum value, (b) when kinetic energy is near its minimum value, and (c) just before liquid bridge separates from bulbous ends. $\mathrm{We}=58$ and $\mathrm{Re}=1127$. Contours are for the $\mathrm{Y}-\mathrm{Z}$ plane at $\mathrm{X}=0$.
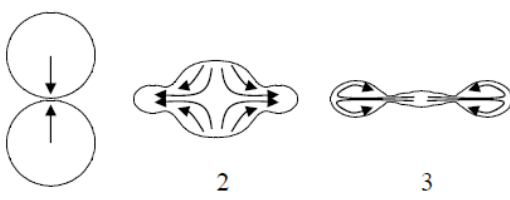

3
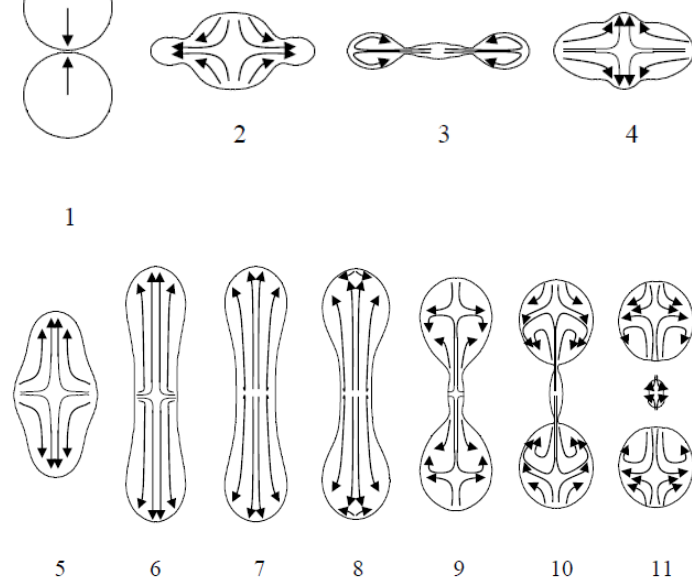
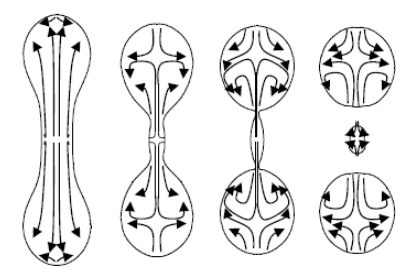

10 torus as observed at the beginning of the second period of radial contraction. It is interesting to note that in the rim of the torus a circulation pattern develops which is due to the combined effect of surface curvature and the high pressure region near the center. Eventually, the torus collapses and the flow is redirected outward (images 4-6). Once the combined mass has stretched to its maximum length, the curvature of the bulbous ends causes the fluid near the surface to be redirected back toward the center of the combined mass as shown in the eighth image on Fig. 2.

At this point, fluid is still flowing outward (causing the contraction which is observed along the radial axis) and collides with the re-directed fluid flowing inward due to the surface curvature. This stagnation region causes the flow to be re-directed radially in the middle of the bulbous ends which in turn is forced inward at the surface due to surface tension. This increases the curvature of the bulbous end and as a result contributes to the observed necking at the attachment region between the ligament and the ends. This flow pattern continues until break up is observed. The final flow pattern in the separated drops, therefore, contains four regions of circulating flows and resembles the internal flow patterns of a drop exposed to an external hyperbolic shear flow.

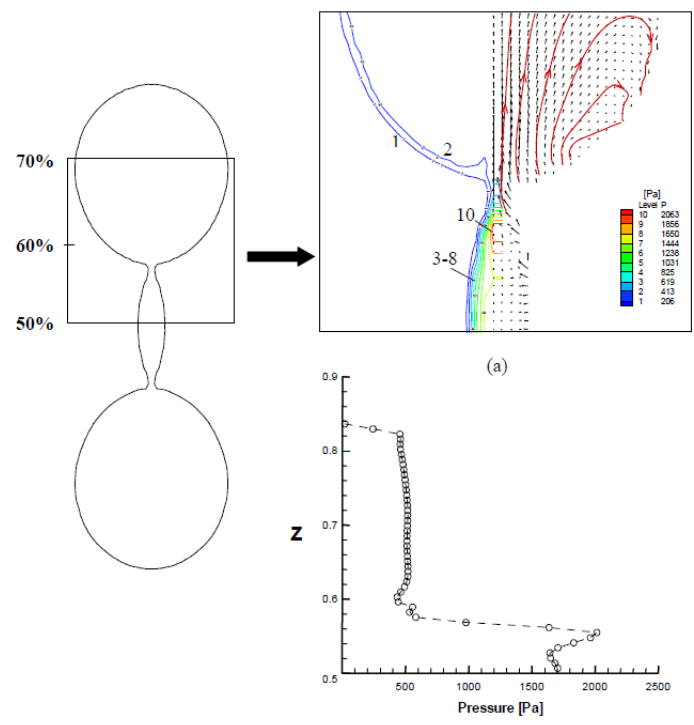

(b)

Fig. 6. Example of Pinching. Result of a $\mathrm{We}=50$ and $\mathrm{Re}=1596$ collision at time equal to $4.24 \mathrm{~ms}$. (a)

Magnified view of pinched region, pressure contours (left) and velocity vectors (right). (b) Pressure variation along the axial direction.

Figure 6 illustrates the details of the breakup for a collision with $W e=50$ and $R e=1596$. Figure 6a contains both the pressure contours and velocity vectors of an enlarged area of the necking region. A companion Fig. 6b, represents the pressure variation along the axial direction. The breakup is initially caused by the presence of two high pressure regions. Curvatures in the radial and lateral directions cause a high pressure region to develop in the ligament. Similarly, another high pressure region develops in the bulbous ends due to its 
curvature. This pressure difference causes more mass to be accumulated in the end region, which results in a negative curvature at the surface where the ligament and bulbous ends are connected. This sets up a minimum pressure which subsequently causes a local decrease in fluid mass and thus the formation of a neck. Once the neck is formed, it helps to increase the pressure, which causes the fluid to recede further causing more "necking". This process continues until the neck becomes so thin that it breaks.

\subsection{Critical Weber Number for the Onset of Separation}

Comparisons of Fig. 1 and 2 show that at some Weber number between $W e=40$ and 58 , there is a critical Weber number, $W e_{c r}$, which marks the boundary between coalescence and separation. Experimentally, Adam et al. (1968) reported that the critical Weber number varies based on the drop size. They reported critical Weber numbers of 60 and 100 for water drops with diameters of $120 \mu \mathrm{m}$ and $600 \mu \mathrm{m}$, respectively. Other experiments reported much smaller critical Weber numbers and no dependency on the droplet diameter.

For example Ashgriz and Poo (1990) determined the critical Weber number for equal sized water drops colliding in atmospheric air to be approximately 19 . This value has been verified by the work of Qian and Law (1997). Additionally, Jiang et al. (1992) reported various lower critical Weber numbers for different hydrocarbons, and therefore demonstrated its dependency on the fluid type. In their work the critical Weber number of tetradecane was found to be 34 , which was subsequently confirmed by Qian and Law (1997).

Table 2 Computed Critical Weber Numbers for Different Fluids and Drop Diameters

\begin{tabular}{|c|c|c|c|c|}
\hline Fluid & Diameter $[\mu \mathrm{m}]$ & $\begin{array}{c}\text { Velocity } \\
{[\mathrm{m} / \mathrm{s}]}\end{array}$ & $W e_{c r}$ & $\operatorname{Re}$ \\
\hline Water & 300 & 3.3 & 45.8 & 1002 \\
\hline Water & 500 & 2.4 & 41.0 & 1223 \\
\hline Water & 700 & 2.0 & 39.1 & 1414 \\
\hline Water & 2000 & 1.1 & 34.4 & 2240 \\
\hline Mercury & 300 & 2.0 & 33.6 & 5000 \\
\hline
\end{tabular}

In this investigation the numerically determined critical Weber number for different drop sizes and fluid types are shown in Table 2. The numerically determined critical We numbers are larger than those reported by Ashgriz and Poo 1990, but less than those reported by Adam et al. (1968). This may be attributed to the experimental technique used for determining the critical We number. In Ashgriz and Poo (1990) two streams of equally sized droplets are aimed at each other with collision angles ranging from 20 to 90 degrees. Therefore, droplets have a forward moving trajectory at the time of collision. Although, it is assumed that only the relative velocity governs the collision outcome, it appears that such experiments in the presence of air result in an internal flow field, which may enhance the onset of separation.
On the other hand, Adam et al. (1968) aimed two droplets directly towards each other $\left(180^{\circ}\right.$ angle $)$. In addition, they used relatively large droplet spacings so that the collisions were not in the wake of the previous ones, as was the case in Ashgriz and Poo (1990). Our simulations are similar to experiments of Adam et al. (1968), however, ours is in a dynamically inert gas. Therefore, there is less resistance for the droplet motion, and thus the critical We numbers are slightly smaller. One important finding is that the droplet size has an influence on the critical We number. This confirms the results of Adam et al. (1968), and indicates that the collision We number is not the only parameter that governs the collision process.

For a wide range of Weber and Reynolds numbers tested here, no reflexive separation is observed for the tetradecane drops. Of the three fluids considered, tetradecane has the lowest surface tension coefficient, $\sim 0.37 \sigma_{\text {water }}$ and $\sim 0.06 \sigma_{\text {mercury }}$, and the largest kinematic viscosity, $\sim 3 v_{\text {water }}$ and $\sim 25 v$ mercury. Both of these fluid properties have a prominent effect on either surface energy or energy dissipation, respectively. In addition, density is also important since for the same collision parameters, it changes the initial kinetic energy. The density of Tetradecane is again the smallest of the three: $\sim 0.76 \rho_{\text {water }}$ and $\sim 0.06 \rho_{\text {mercury }}$. Therefore, tetradecane has the least surface energy, the highest energy dissipation and the least initial kinetic energy as compared to similar collisions of water and mercury drops.

\subsection{Evaluation of Collision Models}

There are two very different types of models for the prediction of the onset of separation in a head-on collision of two drops. One is based on the capillary instability theory, according to Ashgriz and Poo (1990) and Brenn et al. (2001), and the other is based on energy dissipation, according to Jiang et al. (1992), Qian and Law (1997), and Willis and Orme (2004). In this section, we will assess the accuracy of the major assumptions made in these models.

\subsubsection{Capillary Instability Based Models}

Ashgriz and Poo (1990) proposed a capillary instability model to predict the boundary between coalescence and reflexive separation. The underlying principle in their model is the following. When the two drops collide, they first form a thin disk which will later contract into a round-ended cylinder with radius $r$ and length $l+2 r$, as shown in Fig. 7a.

Based on Rayleigh's linear theory (1878), if the lengthto-diameter ratio of a liquid column is greater than or equal to $\pi$, the column becomes unstable. Ashgriz and Poo used the whole length of the round ended drop, $l+2 r$, as the length of the column. Then, by applying the Rayleigh criterion to the round-ended drop of Fig. 7a, they found the instability criterion as $(l+2 r) / 2 r=\pi$. The round ended cylinder is, however, different from a liquid column, which does not have surface forces pushing in from its two ends. For this drop, if the fluid inside is stagnant, the surface forces from the two ends will force the flow towards the center, preventing the 
break-up. Therefore, this liquid cylinder can break only if the internal flow field stops the retraction of the two ends. If the round ended cylindrical drop is held stationary, and disturbances can grow and break the drop. Based on this model, Ashgriz and Poo postulated that (1) if length to diameter of the liquid column at the end of the reflex phase is equal to $\pi$, and (2) if there is enough kinetic energy inside the drop to keep the column stationary, then the drop can break.

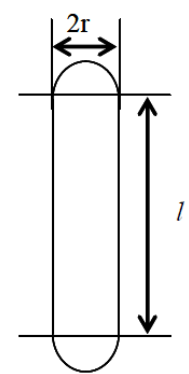

(a)

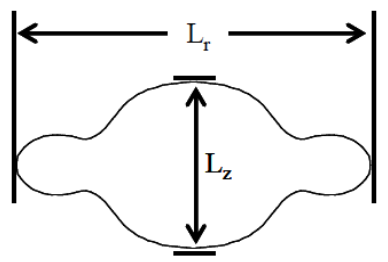

(b)

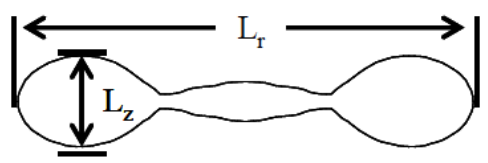

(c)

Fig. 7. (a) A round ended liquid column formed after collision. Ashgriz and Poo (1990) postulated that this column will be unstable if its length $(l+2 r)$ is larger than its perimeter $(2 \pi r)$. (b) and (c) Axial and radial deformation lengths defined as difference between maximum and minimum points in corresponding direction. Early evolution shapes from two $700 \mathrm{~mm}$ water drops colliding at $\mathrm{We}=50$ and $\mathrm{Re}=1596$.

We will first determine the critical length of a combined drop beyond which it will breakup into two or more drops. We will express the extent of deformation as follows:

$\eta=\frac{L_{r}}{D}$

$\psi=\frac{L_{z}}{D}$

where $L_{r}$ and $L_{z}$ are computed by identifying the maximum and minimum points located on the fluid surface on the radial and the axial directions, respectively (Fig. $7 \mathrm{~b}$ and $7 \mathrm{c}$ ), and $D$ is the initial drop diameter. Note that these values are not necessarily along the axes. For instance, the maximum deformation $L_{z}$ can be at the edges of the torus and not at its center. We have chosen the actual maximum and minimum points in order to capture the diameter of the end points of the combined mass.

(a)

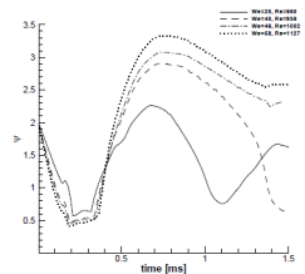

(b)

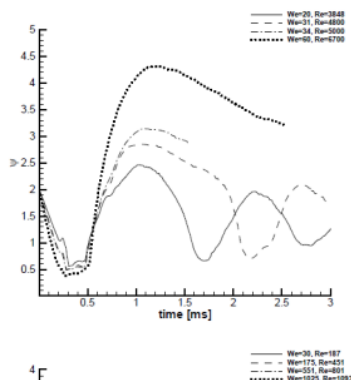

(c)

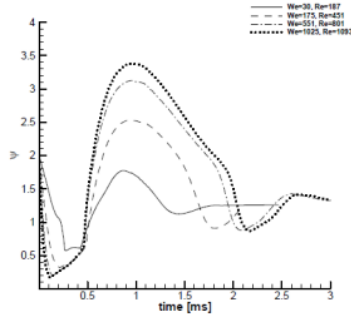

Fig. 8. Extent of Deformation along the axial direction, $\psi=L_{z} / D$, where $L_{z}$ is the distance

between the maximum and minimum points along the axial direction. (a) Water, (b) Mercury and (c)

Tetradecane. $D=300 \mu m$.

Figures 8 and 9 show the time variations of dimensionless axial deformation, $\psi$, and dimensionless radial deformation, $\eta$, respectively, for $300 \mu \mathrm{m}$ drop collisions for water, mercury and tetradecane. These figures show that during the collision phase, $\psi$ reaches a minimum at $Z_{\min }$, while $\eta$ reaches a maximum. For fluids with low surface tension coefficients (i.e., tetradecane), $\psi$ represents the thickness of a flat disk, while for larger surface tension coefficients (i.e., water and mercury), $\psi$ corresponds to the diameter of a torus' outer rim.

Figure 8 shows that once $\psi$ reaches a minimum, it remains around that position for a short time and then it starts to increase. This can be understood by comparing Fig. 8a with Fig. 1 and 2. In Fig. 1 , at $\mathrm{t}=0.15 \mathrm{~ms}$ the height of the drop along the $z$-axis has become almost the same as the drop thickness on the rim of the torus. In the following time steps, the height along the $z$-axis reduces, while the thickness of the rim increases slightly. Once the reflexive action takes over, the droplet height along the $z$-axis increases rapidly, bypassing the rim thickness. This plot allows one to compare the rim thickness for various conditions. A general conclusion is that the diameter of the rim of the torus for the water and mercury drops and for a wide range of conditions shown in Fig. 8 is approximately equal to the initial radius of the drops (i.e., $\psi \approx 0.5$ ). 
(a)

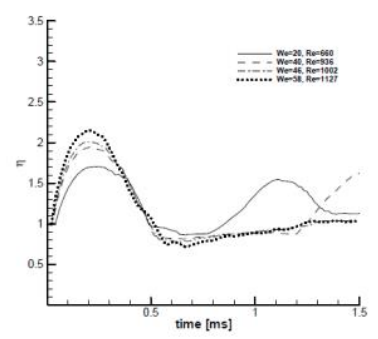

(b)

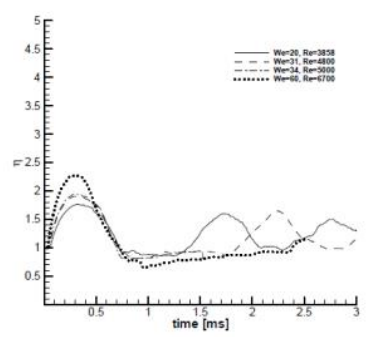

(c)

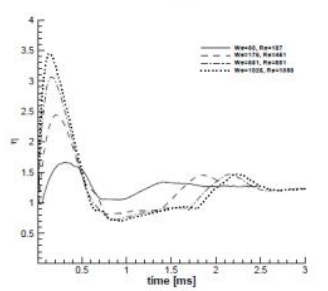

Fig. 9. Extent of Deformation along the radial direction, $\eta=L_{r} / D$, where $L_{r}$ is the distance between the maximum and minimum points along the radial direction. (a) Water, (b) Mercury and (c) Tetradecane. $D=300 \mu \mathrm{m}$.

During the reflexive phase, all three fluids exhibit very similar behavior in the way in which the radial deformation (i.e. contraction) varies with time. This similar behavior is noted due to the deformation curves overlapping one another for water and mercury collisions and is thus independent of Weber number. Similarly, while $\eta$ does not explicitly overlap for tetradecane collisions (owing to the large range in initial fluid impact energy), the change in deformation (i.e. the slope) is relatively the same regardless of Weber number. This indicates that the surface tension forces in the reflexive phase dominate the fluid motion.

Once $\psi \max$ is reached, $\eta$ variations become similar and again independent of Weber number. However, the behavior of deformation in the axial direction is quite different depending upon the collisional outcome. For cases resulting in coalescence, $\psi$ decreases rapidly, indicating the dominance of the inertia forces. As the Weber number increases, the final outcome changes from coalescence to separation and the corresponding $\psi$ exhibits slower changes (smaller slopes) - an indication that inertia and surface forces are competing for control. If surface tension begins to dominate as the combined mass contracts, radial pinching develops and grows, resulting in separation. Conversely, if the axial fluid momentum dominates, radial pinching will not develop, resulting in permanent coalescence instead. Therefore, if the combined mass stretches to a critical maximum length, $\left(\psi_{\max }\right)_{c r}$, the influence of surface tension compared to fluid momentum is now such that

surface tension dominates the collisional evolution and the combined mass will separate.

An important observation from Fig. 8 is that the demarcation point of the coalescence and separation is around $\psi=3.1$ for both water (Fig. 8a) and Mercury (Fig. 8b). However, for the tetradecane drops and for a wide range of Weber numbers tested here, even for much larger $\psi$, droplet separation was not observed.

Therefore, the axial length criterion by itself may not be sufficient to predict the onset of separation. A closer look at the difference between the water, mercury, and tetradecane plots (Figs. 8 and 9) reveals that the liquid column breaks only if a bulbous end at the rim of the collision phase is observed. Comparing Figs. $8 \mathrm{a}$ and $8 \mathrm{~b}$ with Fig. 8c, shows that for water and mercury the minimum $\psi$ stays at about 0.5 or half of the drop initial diameter, yet for tetradecane, it can continuously reduce. This indicates that for tetradecane collisions the collision phase results in the formation of a flat disk and not a torus. Therefore, the surface area at the tip of the rim of the combined drop is small as compared to the surface area of a torus. Therefore, it can be said that a torus-shape at the end of the collision phase with a rim diameter equal to the radius of the initial drops is the second requirement for the onset of separation.

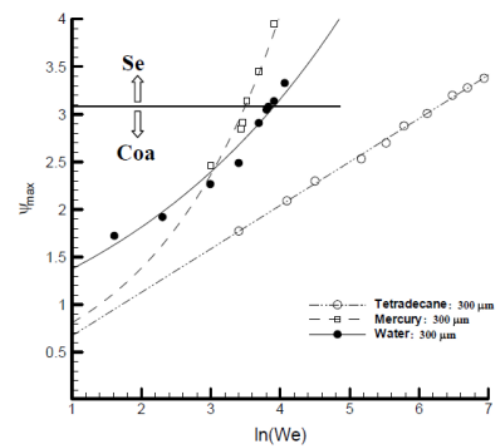

(a)

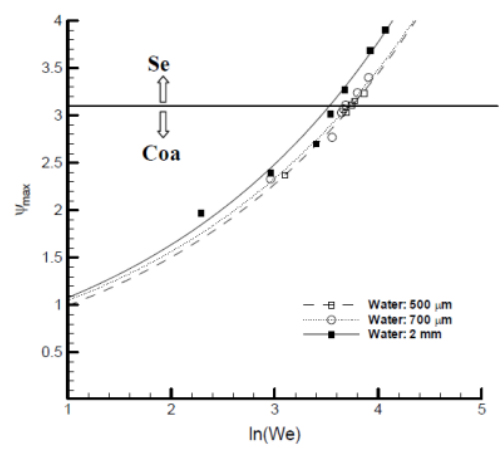

(b)

Fig. 10. Plots of $\psi \max$ versus $\ln (\mathrm{We}$ ) (a) Comparison of different fluids and $D=300 \mu \mathrm{m}$. (b) Comparison of different water drop sizes.

A search for this critical deformation value was accomplished by plotting $\psi \max$ versus $\ln (W e)$ for the three fluid types and different drop sizes. The results are plotted in Fig. 10, where Fig. 10a provides a comparison of fluid types and Fig. 10b gives a 
comparison of drop sizes. Since the range of Weber numbers used in the simulation of tetradecane collisions was an order of magnitude larger than the values used for water and mercury drop collisions, the natural logarithmic scale was used. This scale allows the data to be represented on a single plot and it is useful for comparison purposes.

Review of Fig.10 allows two observations to be made. First, the behavior of collisions composed of water or mercury are very different than the observed variation seen for tetradecane drops. Second, for water and mercury collisions the lower critical Weber numbers of 33.6 and 45.8 indicate that $\left(\psi_{\max }\right)_{c r} \sim \pi$. Therefore, if the combined mass stretches in the axial direction to a value greater than $\pi$, separation will always occur. However, for tetradecane drop collisions, $\left(\psi_{\max }\right)_{\mathrm{cr}}$ does not exist since reflexive separation never occurs because of small rim surface areas during the collision phase. A similar review of Fig. 10b, which represents a comparison with respect to drop size, reveals that size has little impact on this parameter's variation. In all three drop size cases, at the lower critical Weber numbers of 41.0, 39.1 and 34.4 , the critical length to drop diameter ratio is $\left(\psi_{\max }\right)_{\mathrm{cr}} \sim \pi$.

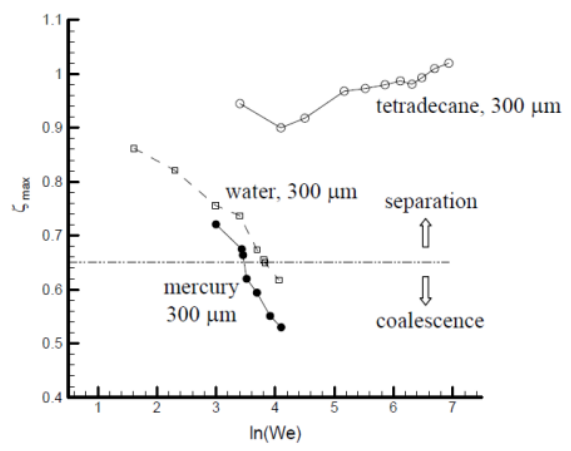

(a)

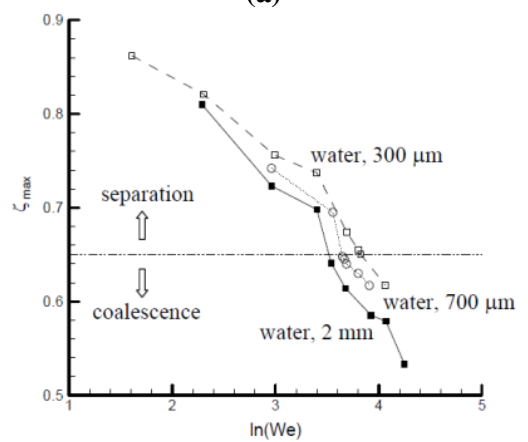

(b)

Fig. 11. Ratio of maximum dimensionless deformations in the radial and axial direction, $\zeta_{\max }=\eta_{\max } / \psi_{\max }$. (a) Comparison of different fluids. (b) Comparison of different water sizes. The dashed line represents the boundary between coalescence and separation.

Another criterion for the collision outcome was observed by plotting the ratio of the maximum dimensionless deformation in the radial and axial directions, $\zeta=\eta_{\max } / \psi \max$. A comparison of this ratio with $\ln (W e)$ for (a) tetradecane, water and mercury and (b) for water drop diameters of $300 \mu \mathrm{m}, 700 \mu \mathrm{m}$ and 2 $\mathrm{mm}$ is provided in Fig. 11. In all cases for water and mercury, a critical value of $(\zeta)_{\mathrm{cr}}=0.65$ represents the demarcation value between permanent coalescence and separation. This ratio for tetradecane collisions always remained above 0.9 and is consistent with the fact that reflexive separation was never observed.

We can now address the capillary instability based criterion for breakup proposed by Ashgriz and Poo (1990). As noted earlier, they assumed that breakup occurs when length to diameter of a round ended drop is more than $\pi$, i.e., $(l+2 r) / 2 r=\pi$. Based on our model, $L_{z}=l+2 r \equiv l+D^{\prime}$ where $D^{\prime}$ is the diameter of the liquid ligament formed after collision. Diameter of the droplet relates to the diameter of the liquid ligament at this critical condition as $D=1.28 D^{\prime}$. Therefore, the critical condition based on our parameters is $(l+2 r) / 2 r \equiv L_{z} / D^{\prime}=\pi$ or $L_{z} / D=\psi=2.45$. This is different than our finding of $\psi=\pi$. If the length of the column in Ashgriz and Poo's model did not include the round ends, then their prediction of the critical conditions would have been $\psi \approx 2.9$. In fact, the Rayleigh instability criterion is for an infinitely long column and not for a round ended column. Therefore, a modified criterion for breakup based on length $l$ as shown in Fig. 7a, better matches the numerical results. This condition is only valid for collisions in which the liquid surface tension is large enough to produce a torus-shape drop during the collision phase.

\subsubsection{Dissipation Based Models}

Jiang et al. (1992) have used a dissipation based model to describe the onset of separation. They assumed that at the time when the combined drop has reached its maximum deformation along the radial axis (i.e., $R_{\max }$ ) there is no internal kinetic energy. All the initial kinetic and surface energies of two drops is transferred partly to the surface energy and the rest is dissipated. Equating the initial total energy to the surface energy plus dissipated energy at this time yields

$K E_{o}+S E_{o}=S \sigma+\phi$

where $S$ is the surface area of the united spheroid and $\phi$ is the dissipated energy at this instant in time. By nondimensionalizing with respect to the initial surface energy, they derived the following relationship,

$\hat{S}=1+(1-\varphi) W e^{*}$

where $\varphi$ is the dissipation coefficient. The limiting case is $\varphi=0$. By using their experimental data, they concluded that $\varphi=0.5$ which indicates that half of the initial energy is dissipated during this phase of the evolution and that its value is independent of the viscosity of the drop. We will show that the dissipation energy at the end of the collision phase is not half of the initial surface energy, and that the combined drop at this phase has a significant amount of kinetic energy stored in it. Qian and Law (1997) expanded this model to include dissipation losses associated with all phases of the evolution sequence. In particular, they assumed that in order for separation to occur, the initial kinetic 
energy must be larger than all losses due to dissipation and any residual energy associated with the deformed shape of the combined mass. This relationship is represented as

$$
K E_{o}=\phi_{1}+\phi_{2}+\phi_{3}+\Delta S \sigma
$$

where $\phi_{1}$ represents the dissipation losses associated with the collision phase, $\phi_{2}$ those with the reflexive phase, and $\phi_{3}$ those with separation or oscillation phases. $\phi_{l}$ was estimated using the $\varphi=0.5$ as indicated by Jiang et al. (1992), while $\phi_{2}$ and $\phi_{3}$ were estimated by using both geometrical relations and estimates for the characteristic velocity during each phase. Substituting and simplifying, they obtained the following expression for the computation of the critical Weber number

$$
W e_{c r}=\beta \cdot O h^{*}+\gamma
$$

where $\beta$ is a constant and a function of geometry and the dissipation coefficient, $\varphi$, and $O h^{*}=16 \mu / \sqrt{\rho R \sigma}$ is a modified Ohnesorge number. The parameter, $\gamma$, represents the additional surface energy needed for separation and is also a function of $\varphi$. Based upon their experimental work, they concluded that $\beta=24 \pm 3$ and $\gamma=10 \pm 2$, which correlated nicely with a fitted line represented by $W e_{c r}=30 Z+15$. In their work, although viscosity was incorporated into expressions for $\phi_{2}$ and $\phi_{3}$, viscosity was still seen to have a negligible effect upon the outcome.

The first issue, we would like to address is, whether the dissipation assumptions made in Jiang et al. (1992), as well as Qian and Law (1997) are plausible. In order to determine the energy dissipation, we will determine the evolution of the surface energy, the kinetic energy and the dissipation in the colliding drops. At the beginning of the collision, both drops have a known amount of surface and kinetic energy. These energies are computed as follows

$$
\begin{aligned}
& S E_{o}=2 \pi D_{i}^{2} \sigma \\
& K E_{o}=\rho \frac{\pi}{24} D_{i}^{3} V_{r}^{2}
\end{aligned}
$$

where the ratio of this kinetic energy to surface energy is defined to be $\mathrm{We}^{*}=\mathrm{We} / 48$ (Jiang et al. 1992). Numerically, at each time step, each of these energies are computed using the following definitions

$$
\begin{aligned}
& S E_{n}=\sigma \sum_{p=1}^{N_{\text {polygons }}} S A_{p} \\
& K E_{n}=\frac{1}{2} \sum_{i j k=1}^{N_{\text {cells }}}\left(\frac{f_{i j k} \rho}{v_{\text {cell }}}\right)\left(\mathrm{u}_{i j k}^{2}+\mathrm{v}_{i j k}^{2}+\mathrm{w}_{i j k}^{2}\right)
\end{aligned}
$$

where $S A_{p}$ is the polygon area and $v_{\text {cell }}$ is the volume of the computational cell. Therefore, using these relationships each energy variation was computed at each time step during the collisional process. Additionally, by using these energies and the conservation of energy equation an estimation of the change in total energy or dissipation energy, $\phi$, was obtained as follows

$\phi=K E_{o}+S E_{o}-K E_{n}-S E_{n}$

We will scale the kinetic and surface energies with the initial surface energy as

$$
\begin{gathered}
\alpha=\frac{K E_{n}}{S E_{o}} \\
\chi=\frac{S E_{n}}{S E_{o}}
\end{gathered}
$$

The initial surface energy was chosen as the scaling energy since for a same size and same fluid drop collisions, this energy is independent of impact energy. Since the amount of dissipated energy is simply the combined transient kinetic and surface energies subtracted from the initial total energy, a nondimensional dissipation energy was defined as

$\Phi=1-\left(\frac{K E_{n}+S E_{n}}{K E_{o}+S E_{o}}\right)$

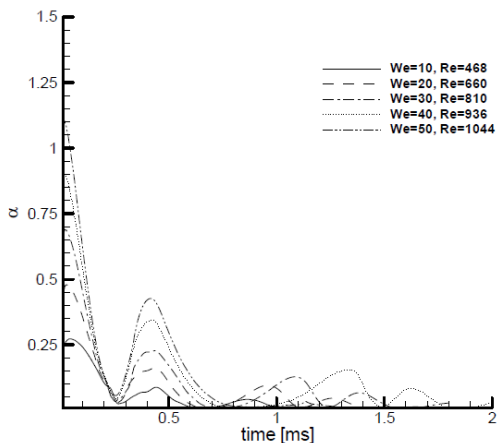

(a)

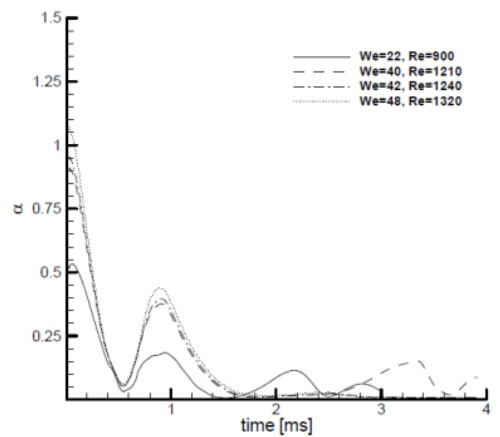

(b)

Fig. 12. Dimensionless kinetic energy, $\alpha=K E_{n} / S E_{o}$ versus time. (a) Water, $300 \mu \mathrm{m}$ and (b) Water, $500 \mu \mathrm{m}$.

\subsubsection{Kinetic Energy}

Dimensionless kinetic energy variations for $300 \mu \mathrm{m}$ tetradecane, $300 \mu \mathrm{m}$ mercury and $300 \mu \mathrm{m}, 500 \mu \mathrm{m}, 700$ $\mu \mathrm{m}$ and $2 \mathrm{~mm}$ water drops collisions are provided in Fig. 12. In these cases, as the drops come into contact with one another (i.e. time of collision $=0$ ), initially there is a spike in the kinetic energy. As an example, two $300 \mu \mathrm{m}$ water drops colliding with a We/Re combination of $10 / 468$ and 50/1044, respectively, resulted in a relative increase of $31 \%$ and $8 \%$. Similar behavior was observed for other sizes and fluid types. 
This increase occurs very quickly, usually in less than 150 time steps (i.e., less than $0.04 \mathrm{~ms}$ ) and decreases with increase of the We/Re combination. This increase is due to the high pressure region which results from the perpendicular and opposing flow of the two colliding drops. This high pressure area causes the fluid to accelerate radially away from the center thus causing the observed increase in kinetic energy. Immediately afterwards, the kinetic energy decreases transferring energy to the surface energy. At the end of the collision phase, the total kinetic energy is at its minimum value. A maximum kinetic energy is observed between $t_{1}<t_{k e}$ $<\mathrm{t}_{2}$ as defined in Fig. 3, and its value is relatively independent of Weber number. For mercury drop collisions with a lower critical Weber number of 33.6, this maximum ratio of kinetic energy to initial surface energy $\alpha_{\max }=0.368$. For water drop collisions with drop diameters of $300 \mu \mathrm{m}, 500 \mu \mathrm{m}, 700 \mu \mathrm{m}$ and $2 \mathrm{~mm}$, this ratio is $0.401,0.396,0.380$ and 0.353 , respectively. Therefore, for mercury and water drop collisions, the maximum amount of kinetic energy for separation can be approximated by computing $37 \%$ of the initial surface energy. It should be noted that at the end of the collision phase the kinetic energy is very small (but not zero), and therefore, Jiang et al. (1992) assumption that the flow comes to a stop at that point is a good approximation.

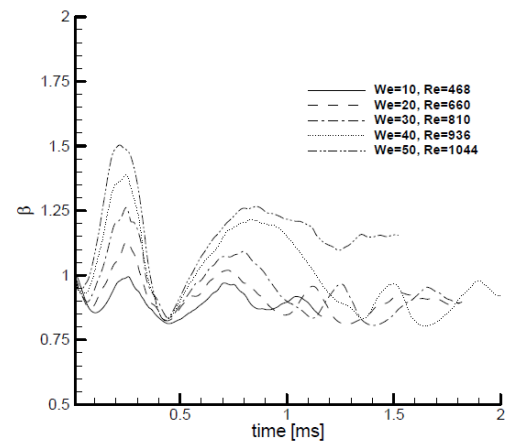

(a)

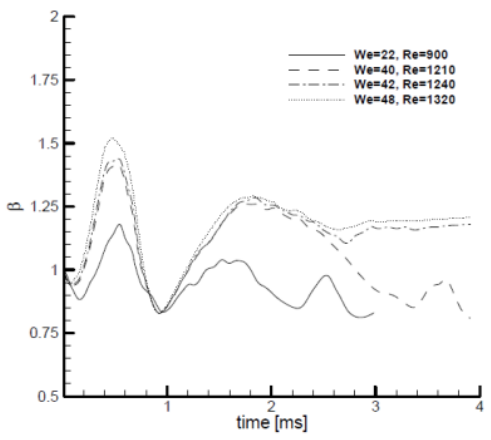

(b)

Fig. 13. Dimensionless surface energy, $\beta=S E_{n} / S E_{o}$ versus time. (a) Water, $300 \mu \mathrm{m}$ and (b) Water, $500 \mu \mathrm{m}$.

\subsubsection{Surface Energy}

As with the previous discussion on kinetic energy, the dimensionless surface energy as a function of time is plotted in Fig. 13. As expected, the maximum surface energy occurs at the end of the collision phase where the combined fluid has spread along the radial axis to its maximum radius, $R_{\max }$. For mercury drop collisions, the maximum dimensionless surface energy at the transition point between coalescence and separation is $\chi_{\max }=1.43$. For water drop collisions with drop diameters of $300 \mu \mathrm{m}, 500 \mu \mathrm{m}, 700 \mu \mathrm{m}$ and $2 \mathrm{~mm}$, this ratio is $1.46,1.44,1.43$ and 1.40 , respectively. Taking the averages yields $\bar{\chi}_{\max }=1.433$, which is very close to mercury's value of 1.425 . Therefore, the maximum amount of surface energy for separation can be computed by taking 1.43 times the initial surface energy. For collisions composed of tetradecane, this ratio varied from 1.03 to 3.20 for Weber numbers of 30 to 1025 , respectively. Although $\chi_{\max }=1.45$ occurs for a Weber number of 90 , no separation is observed. It is concluded that models which are based only on the surface energy at a certain point are not by themselves appropriate to define the onset of separation.

\subsubsection{Dissipated Energy}

Two aspects of the Jiang et al. (1992) model are tested using the numerical results obtained in this investigation. First, the overall form of Eq. (11) is tested. This was done by computing the modified Ohnesorge number, $\mathrm{Oh}^{*}$, for water drop collisions with diameters of $300 \mu \mathrm{m}, 500 \mu \mathrm{m}, 700 \mu \mathrm{m}$ and $2 \mathrm{~mm}$ and using their corresponding lower critical Weber number. The results of this analysis along with the results provided by Qian and Law (1997) are plotted on Fig. 14a. Mercury is also included since it was observed that reflexive separation occurred at $W e_{c r}=33.6$. A linear line fitted through the water data provides, $W e_{c r}=116(\mathrm{Oh} *)+28$. This should be compared with that of Qian and Law's empirical relation of $W e_{c r}=30\left(O h^{*}\right)+15$. Therefore, in Eq. 11, $\beta_{\text {num }}=116 \sim 3.9 \beta_{\exp }$ and $\gamma_{\text {num }}=28 \sim 1.9 \gamma_{\text {exp }}$, where the subscripts exp and num refer to experimentally and numerically determined constants. Both of these numerical constants indicate that in a vacuum environment, collisions result in more substantial changes in geometry (i.e. deformation) than for collisions occurring in a gaseous environment, and/or less dissipation occurs during the collision phase. This is, in fact, observed in the experiments of Willis and Orme (2000).

The second aspect tested is related to the issue concerning the amount of dissipation occurring in the initial phase of the evolution. Jiang et al. (1992) define this dissipation coefficient to be

$$
\varphi=\frac{\phi_{1}}{S E_{o} W e^{*}}
$$

By substituting the amount of dissipation occurring during the collision phase and the initial surface energy and the modified Weber number for water drop collisions of $300 \mu \mathrm{m}$ and $2 \mu \mathrm{mm}$ colliding at their corresponding critical Weber number, this coefficient is 0.389 and 0.299, respectively. Qian and Law (1997) derived expressions for $\beta$ and $\gamma$, which represent the product of two factors. Specifically, both are functions of the dissipation coefficient, while $\beta$ is also a function of the geometry and $\gamma$ is also a function of excess surface energy. In general, each of these constants can be written as follows: 
$\beta=C_{\text {dissipation }} C_{\text {geometry }}$

$\gamma=C_{\text {dissipatim }} C_{\text {surface }}$

Here $C_{\text {dissipation }}=1 /(1-\varphi), C_{\text {geometry }}=f($ geometry $)$ and $C_{\text {surface }}=6 \Delta S / \pi R^{2}$. Therefore, using the original Jiang et $a l$. assertion that $50 \%$ of the energy is dissipated and Qian and Law's values of $\beta_{\exp }=30$ and $\gamma_{\exp }=15$, the factors associated with geometry and excess surface energy can be estimated. Doing so yields $C_{\text {geometry }}=15$ and $C_{\text {surface }}=7.5$.

Now, using these constant values and an average dissipation coefficient based upon the results obtained in this investigation, $\bar{\varphi}=0.348, \beta$ and $\gamma$ are recomputed to be $\beta^{\prime}=23.0$ and $\gamma^{\prime}=11.5$. Although these values more closely approximate Qian and Law's (1997) experimental determination of $\beta=24 \pm 3$ and $\gamma=10 \pm 2$, they do not agree with the plotted data of $\beta_{\text {num }}=116$ and $\gamma_{\text {num }}=28$. This again supports the assertion that for collisions in a vacuum, more pronounced deformation will occur as compared to collisions occurring in the presence of a surrounding gaseous environment.

Willis and Orme (2000) presented a model based upon their experimental observations of oil drop collisions in a vacuum. Using Jiang et al.'s (1992) equation for total energy at the instant of maximum spread along the radial axis, they proposed that the amount of surface energy can be bounded by two limiting shapes. Namely, these shapes are either a thin flat disk, or the minimum surface area, $S_{d}$, and a torus, representing the maximum surface area, $S_{t}$. Each of these areas are computed using analytical expressions and geometric values taken from their experimental data. Non-dimensionalizing with respect to the initial surface area of two spherical drops, an average surface area is computed as follows

$\hat{S}=\frac{\hat{S}_{t}+\hat{S}_{d}}{2} \pm \frac{\hat{S}_{t}-\hat{S}_{d}}{2}$

Using data obtained during this investigation, this variable was computed and plotted versus the scaled Weber number, We* for tetradecane, water and mercury drop collisions. The results of which are provided in Fig. 14b. Additionally, Eq. (9) is provided for the case when there is no dissipation and when the dissipation coefficient is $50 \%$. Willis and Orme provided a similar plot using their experimental data, which indicates that the amount of dissipation is dependent on the fluid viscosity contradicting the original assertion by Jiang et al. In particular, Willis and Orme demonstrated that the amount of dissipation increases as the viscosity of the fluid increases.

Review of Fig. 14b supports this assertion, since tetradecane, water and mercury all exhibit independent behavior with regards to one another and as the viscosity of the fluid increases, more energy loss occurs. In fact, increased dissipation causes the overall behavior to become more nonlinear (i.e., tetradecane versus mercury and water). Additionally, for the three fluids investigated it is observed that as $\mathrm{We}^{*} \rightarrow 0$, the non-dimensional surface area also approaches a limit, namely $\hat{S} \rightarrow 2^{-1 / 3}$. At low Weber numbers, the final outcome of droplet collisions is permanent coalescence. This limiting value is computed by calculating the surface area of the final combined droplet divided by the total surface area of the two original drops.

(a)

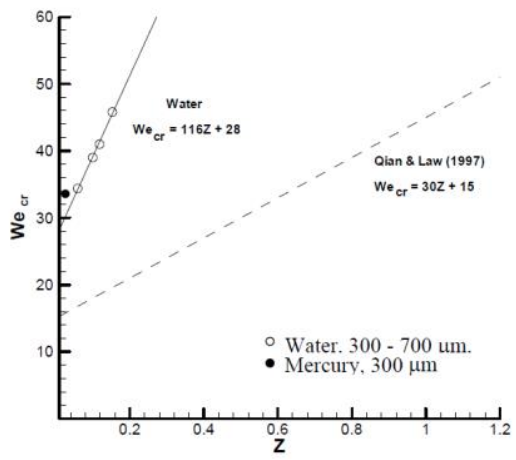

(b)

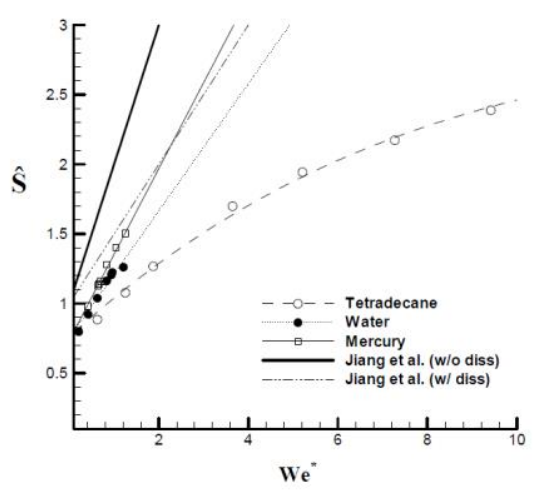

Fig. 14. Model Comparison. (a) Qian and Law Model (b) Willis and Orme model compared with Jiang et al. Model

\subsection{Reynolds Number Effect}

There has been much speculation as to whether the Reynolds number has an effect or not on the overall collisional outcome. In this section, this aspect was tested by simulating collisions with a constant Weber number and various corresponding Reynolds numbers. The appropriate Weber/Reynolds number combination was obtained by either varying the drop diameter and keeping the fluid properties and relative velocity constant or by keeping the drop diameter, relative velocity, surface tension and density constant and varying the kinematic viscosity. In these two ways, the Weber number remains constant while the Reynolds number varies. For instance, an investigation for a constant Weber number of 40 was conducted. In order to simulate a variety of collisions with this Weber number, drops with water properties and diameters of $300 \mu \mathrm{m}, 500 \mu \mathrm{m}, 700 \mu \mathrm{m}$ and $2 \mathrm{~mm}$ were used. These four cases resulted in corresponding Reynolds numbers of 936, 1210, 1428 and 2400 , respectively. Two additional Reynolds numbers of 1040 and 1337 were simulated by defining water drops with the same relative velocity used for the case of $\mathrm{Re}=936$, but values of $0.9 \times 10^{-6} \mathrm{~m}^{2} / \mathrm{s}$ and $0.7 \times 10^{-6} \mathrm{~m}^{2} / \mathrm{s}$ were used to represent the kinematic viscosity.

Nobari et al. (1996) plotted the Weber number versus Reynolds number for ten different binary drop collision 
simulations. Based upon their computational data and the experimental data provided by Jiang et al. (1992), they provided an extrapolation for low Reynolds number cases which represents the boundary between coalescence and separation. In their numerical technique the boundary between colliding drops had to be artificially ruptured before merging could take place. Although experimental data of this nature (i.e. rupture times) do not exist, which undermines the veracity of the data, this work is the first to indicate that a clear boundary or critical Reynolds number does exist.

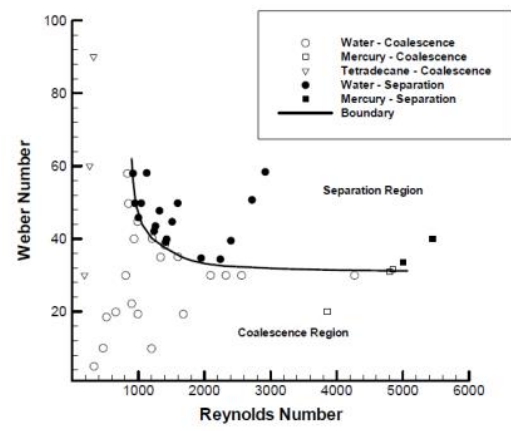

Fig. 15. Weber versus Reynolds Number. Results for Water, Mercury and Tetradecane. Boundary is a fitted spline and represents the transition between coalescence and separation.

Figure 15 shows the critical Reynolds number for the boundary between coalescence and separation obtained from the simulations conducted in this investigation. Review of this data indicates that, as the Weber number increases, a minimum Reynolds number of slightly less than 1000 is required before separation will occur. This value is an order of magnitude greater than the extrapolated value indicated by Nobari, et al. (1996). Additionally, they reported that, as the Reynolds number increases, the Weber number decreases until a limiting value of $W e=24$ is reached. This value of 24 was based upon the work conducted by Ashgriz and Poo (1990) for high Reynolds numbers. In contrast, the results obtained in this investigation indicate a limiting Weber number of 35 .

\section{COnClusions}

Full three dimensional numerical simulations of headon binary drop collisions consisting of either water, mercury or tetradecane were conducted. In order to simulate the evolution of the surface deformation during collision, the volume-of-fluid technique was utilized in conjunction with the Continuum Surface Force method for surface tension effects. During the course of this investigation it is noted that for drops without the influence of the surrounding environment, the lower critical Weber number for water drops are somewhat larger than that observed experimentally.

Additionally, the critical Weber number was seen to be a function of drop size. A critical Reynolds number of about 1000 was determined. For all cases simulated in this investigation, separation occurs if the stretching length is greater than $\pi$ times the drop's initial diameter. This is close to the modified capillary based model of
Ashgriz and Poo (1990), in which the liquid column length does not include the round ended parts of the combined drop. Our number is slightly larger than the critical number of 2.85 predicted by numerical simulation of Morozumi et al. (2005).

This difference may be partially due to the different numerical techniques used and their numerical dissipation levels. It is also noted that a critical length model is not sufficient for the prediction of the separation point. For liquids with a small surface tension, a disk shape drop, rather than a torus-shape drop, is formed at the end of the collision or spread phase. The formation of the torus-shape drop was another condition that had to be satisfied to have separation.

During the collision phase (radial expansion), 13-19\% of the energy is dissipated for water drops, while only $9 \%$ was dissipated for mercury drop collisions occurring at the critical Weber number. The dissipation energy for tetradecane was $36 \%-84 \%$ for collisions ranging from $30 \leq \mathrm{We} \leq 1025$. Therefore, the model of Jiang et al. (1992) which uses a 50\% dissipation for all fluids, is a good assumption for most fuel drops with low surface tension, however, it overestimates the dissipation for water and mercury drops. The Jiang et al. (1992) dissipation coefficient was computed for each fluid type and was seen to vary with both fluid type and drop size. Review of the model by Qian and Law (1997) and the one proposed by Willis and Orme (2000) indicates that the collision outcome is indeed dependent upon viscosity. Finally, the assumption that the internal kinetic energy at the end of the collision phase (i.e., when the two drops collide and spread forming a disk or a torus drop) is approximately zero (Jiang et al. 1992; Willis and More 2000) is a good one for drops studies in this work.

\section{REFERENCES}

Adam, J. R., N.R. Lindbald and C.D. Hendricks (1968). The Collision, Coalescence, and Disruption of Water Droplets. J. Appl. Phys. 39, 5173-5180.

Arkhipov, V.A., I.M. Vasenin and V.F. Trofimov (1983). Stability of Colliding Drops of Ideal Liquid Tomsk: translated from Zhurnal Prikladnoi Mekhaniki i Tekhnicheskoi Fiziki 3, 95-98.

Ashgriz, N. and P. Givi (1987). Binary Collision Dynamics of Fuel Droplets. Int. J. Heat and Fluid Flow 8, 205-210.

Ashgriz, N. and P. Givi (1989). Coalescence Efficiencies of Fuel Droplets in Binary Collisions. Int. Comm. Heat Mass Transfer 16, 11-20.

Ashgriz, N. and J.Y. Poo (1990). Coalescence and Separation in Binary Collisions of Liquid Drops. $J$. Fluid Mech. 221, 183-204.

Ashgriz, N. and J.Y. Poo (1990). FLAIR: Flux LineSegment Model for Advection and Interface Reconstruction. J. Comp. Phys. 93, 449-468. 
Baldessari, F. and L.G. Leal (2006). Effect of Overall Drop Deformation on Flow-Induced Coalescence at Low Capillary Numbers. Phys. Fluids 18(013602)

Brackbill, J.U., D.B. Kothe and C. Zemach (1992). A Continuum Method for Modeling Surface Tension. J. Comp. Phys. 100, 335-354.

Bradley, S.G. and C.D. Stow (1978). Collisions between Liquid Drops. Phil. Trans. Roy. Soc. London 287(A-1349), 635-678.

Bradley, S.G., and C.D. Stow (1979). On the Production of Satellite Droplets during Collisions between Water Drops Falling in Still Air. J. Atm. Sci. 36, 494-500.

Brazier-Smith, P.R., S.G. Jennings and J. Latham (1972). The Interaction of Falling Water Drops: Coalescence. Proc. Roy. Soc. London A-326, 393408 .

Brenn, G. and A. Frohn (1989). Collisions and Merging of Two Equal Droplets of Propanol. Exp. Fluids 7 , 441-446.

Brenn, G., St. Kalenderski and I. Ivanov (1997). Investigation of the Stochastic Collisions of Drops Produced by Rayleigh Breakup of Two Laminar Liquid Jets. Phys. Fluids 9, 349-364.

Brenn, G., D. Valkovska and K.D. Danov (2001). The Formation of Satellite Droplets by Unstable Binary Drop Collisions. Phys. Fluids 13, 2463-2477.

Bussmann, M., J. Mostaghimi and S. Chandra (1999). On a Three-Dimensional Volume Tracking Model of Droplet Impact. Phys. Fluids 11, 1406-1417.

Estrade, J.P., H. Carentz, G. Lavergne and Y. Biscos (1999). Experimental Investigation of Dynamic Binary Collision of Ethanol Droplets - A Model for Droplet Coalescence and Bouncing. International Journal of Heat and Fluid Flow 20, 486-491.

Gao, T.C., R.H. Chen, J.Y. Pu and T.H. Lin (2005). Collision between an Ethanol Drop and a Water Drop. Experiments in Fluids 38, 731-738.

Guido, S. and M. Simeone (1998). Binary Collision of Drops in Simple Shear Flow by Computer-assisted Video Optical Microscopy. J. Fluid Mech. 357, 120 .

Gunn, R. (1965). Collision Characteristics of Freely Falling Water Drops. Science 150, 695-701.

$\mathrm{Hu}$, Y.T., D.J. Pine and L.G. Leal (2000). Drop Deformation, Breakup, and Coalescence with Compatibilizer. Phys. Fluids 12, 484-489.

Hsu, C.F. and N. Ashgriz (2004). Impaction of a Droplet on an Orifice Plate. Physics of Fluids 16, 400-411.
Hsu, C.F. and N. Ashgriz (2004). Nonlinear Penetration of Liquid Drops into Capillaries. Journal of Colloid and Interface Science 270, 146-162.

Jayaratne, O.W. and B.J. Mason (1964). The Coalescence and Bouncing of Water Drops at an Air/Water Interface. Proc. Roy. Soc. London A280, 545-565.

Jiang, Y.J., A. Umemura and C.K. Law (1992). An Experimental Investigation of the Collision Behavior of Hydrocarbon Droplets. J. Fluid Mech. 234, 171-190.

Ko, G.H. and H.S. Ryou (2005). Modeling of Drop Collision-Induced Breakup Process. Int. J. Multiphase Flow 31, 723-738.

Kothe, D.B., R.C. Mjolsness and M.J. Torrey (1991). RIPPLE: A Computer Program for Incompressible Flows with Free Surfaces. LA-12007-MS, Los Alamos National Laboratory.

Lafaurie, B., C. Nardone, R. Scardovelli, S. Zaleski and G. Zanetti (1994). Modeling Merging and Fragmentation in Multiphase Flows with SURFER. J. Comp. Phys. 113, 134-147.

Leal, L.G. (2004). Flow Induced Coalescence of Drops in a Viscous Fluid. Phys. Fluids 16, 1833-1851.

Lord Rayleigh, (1878). On the Instability of Jets. Proc. Lond. Math. Soc. 10, 4-13.

Low, T.B. and R. List (1982). Collision, Coalescence and Breakup of Rain Drops. J. Atm. Sci. 39, 15911606.

Low, T.B. and R. List (1982). Collision, Coalescence and Breakup of Raindrops Part II: Parameterization of Fragment Size Distribution. $J$. Atm. Sci. 39, 1607-1618.

Mashayek, F. and N. Ashgriz (1995). A Spine-Flux Method for Simulating Free Surface Flows. J. Comp. Phys. 122, 367-379.

Mashayek, F., N. Ashgriz, W.J. Minkowycz and B. Shotorban (2003). Coalescence Collision of Liquid Drops. International Journal of Heat and Mass Transfer 46, 77-89.

Menchaca-Roca, A., F. Huidobro, A. MartinezDavalos, K. Michaelian, A. Perez, V. Rodriguez and N. Carjan (1997). Coalescence and Fragmentation of Colliding Mercury Drops. $J$. Fluid Mech. 346, 291-318.

Morozumi, Y., H. Ishizuka and J. Fukai (2005). Criterion between Permanent Coalescence and Separation for Head-on Binary Droplet Collision. Atom. and Sprays 15, 61-80.

Nikolopoulos, N., K. Nikas and G. Bergeles (2009). A Numerical Investigation of Central Binary Collision of Drops. Comput. Fluids 38, 1191-1202. 
M.D. Saroka et al. / JAFM, Vol. 5, No. 1, pp. 23-37, 2012.

Nobari, M.R.H., Y.J. Jan and G. Tryggvason (1996). Head-On Collisions of Drops - a Numerical Investigation. Phys. Fluids 8, 29-42.

Orme, M. (1997). Experiments on Droplet Collisions, Bounce, Coalescence and Disruption. Prog. Energy Comb. Sci. 23, 65-79.

Park, R.W. (1970). Behavior of Water Drops Colliding in Humid Nitrogen. Ph.D. Thesis, The University of Wisconsin, Wisconsin, USA.

Poo, J.Y. and N. Ashgriz (1990). Numerical Simulation of Capillary Driven Viscous Flows in Liquid Drops and Flims by an Interface Reconstruction Scheme. Proceedings of $3 r d$ Int. Colloquium on Drops and Bubbles, Monterey, CA, USA.

Post, S.L. and J. Abraham (2002). Modeling the Outcome of Drop-Drop Collisions in Diesel Sprays. Int. J. Multiphase Flow 28, 997-1019.

Qian, J. and C.K. Law (1997). Regimes of Coalescence and Separation in Droplet Collision. J. Fluid Mech. 331, 59-80.

Rieber, M. and A. Frohn (1995). Three-dimensional Davier-Stokes Simulation of Binary Collisions Between Droplets of Equal Size. J. Aerosol Sci. 26, suppl. 1, S929-S930.

Roisman, I.V. (2004). Dynamics of Inertia Dominated Binary Drop Collisions. Phys. Fluids 16, 34383449.

Ryley, D.J. and B.N. Bennett-Cowell (1967). The Collision Behavior of Steam-Borne Water Drops. Int. J. Mech. Sci. 9, 817-833.

Schelkle, M., M. Rieber and A. Frohn (1996, July). Comparison of Lattice Boltzmann and NavierStokes Simulations of Three-Dimensional Free Surface Flows. FED - 1996 Fluids Eng. Div. Conf., San Diego, CA 236, 207-212.

Unverdi, S.O. and G. Tryggvason (1992). A FrontTracking Method for Viscous, Incompressible, Multi-Fluid Flows. J. Comp. Phys. 100, 25-37.

Whelpdale, D.M. and R. List (1971). The Coalescence Process in Raindrop Growth. J. Geophys. Res. 76, 2836-2856.

Willis, K. D., and Orme, M., 2000. Viscous Oil Droplet Collisions in a Vacuum. Exp. Fluids. 29, 347-358.

Willis, K.D. and M. Orme (2003). Binary Droplet Collisions in a Vacuum Environment: an Experimental Investigation of the Role of Viscosity. Exp. Fluids 34, 28-41.

Yoon, Y., M. Borrell and C.C. Park (2005). Viscosity Ratio Effects on the Coalescence of Two Equal-
Sized Drops in a Two-Dimensional Linear Flow. $J$. Fluid Mech. 525, 355-379.

Youngs, D.L. (1984). An Interface Tracking Method for a 3D Eulerian Hydrodynamics Code. Technical Report 44/92/35, AWRE. 